\title{
Perception of radial distance as a function of magnification and truncation of depicted spatial layout
}

\author{
ERNEST A. LUMSDEN \\ University of North Carolina, Greensboro, North Carolina
}

\begin{abstract}
A $2 \times 2$ factorial design requiring judgments of interobject distance was utilized which separated the effects of magnification per se from the concomitant truncation of the visual field normally effected by optically produced magnification. Only the main effect of magnification was significant, and this was much less than the decreases optically specified by the decreased perspective and texture gradients.
\end{abstract}

It has long been known that when an optic array reflected from a spatial layout is magnified, one experiences some distortion in the perception of the radial distance between objects, the slant and shape of the surfaces of these objects, and the slant of the ground itself. Magnification of an optic array is produced by uniformly transforming all visual angles in that optic array by a factor greater than 1 . When a picture is taken with a normal ${ }^{1}$ lens and viewed from the proper station point for receiving the geometric center of the projection, there is no magnification. The proper station point is perpendicular to the center of the picture plane and at a distance equal to the product of the focal length of the lens utilized in taking the picture and the ratio of the diagonal of the enlarged projection on the picture plane to the diagonal of the slide or photographic film. However, if the picture is viewed from a position closer than the proper station point, the array reflected from the surface of the picture plane will be magnified by a factor equal to the proper viewing distance divided by the closer viewing distance. The effects of magnification produced by this means upon the perception of distance within the depicted spatial layout has been investigated by several investigators over the last 20 years (Bartley \& Adair, 1959; Rosinski, 1974; Rosinski \& Farber, 1980; Smith, 1958; Smith \& Gruber, 1958). It is important for our purposes to note, however, that when magnification is produced by this means, no further truncation of the visual field is effected coincident with the process of magnification. However, because it does not confound the decreased den-

I wish to express appreciation for the invaluable assistance of Patti Budman, a graduate student, in the final editing of this manuscript for publication. Support for preparation for publication was provided by the Graduate Research Council of the University of North Carolina at Greensboro. My mailing address is: Department of Psychology, University of North Carolina, Greensboro, North Carolina 27412. sity of gradients with further truncation, I believe this approach does serve to rather unequivocally demonstrate the relevance of the density of gradients of size, texture, and perspective for the environmental perception of size, distance, and slant of surfaces within a spatial layout. Obviously, when such a demonstration is the purpose of the investigation, then, in order to minimize the likelihood of revealing the planimetric nature of the source of structured light that specifies the spatial layout, only monocular viewing should be utilized. On the other hand, if the purpose is to determine the relevance of the density of these gradients under the usual conditions of viewing pictures, then binocular viewing must be permitted.

The other, more familiar method of magnification involves the use of an optical magnifying lens system such as a telephoto or zoom lens or a telescope. When a picture is taken with a magnifying lens (that is, one having a focal length longer than the diagonal of the exposed film surface), then all visual angles in the optic array are uniformly enlarged by a factor equal to the ratio of the focal length of the magnifying lens to the focal length of a normal lens. Consequently, the visual field that can be transmitted is reduced to the reciprocal of the power of magnification. For example, a 4-power telephoto lens (200-mm focal length in a 35-mm SLR camera) will increase the size of all visual angles in the projected array by a factor of 4 and include only one-fourth of the visual field that a normal lens would have transmitted. Nevertheless, if a picture taken with a 4-power magnifying lens in this manner is viewed from a distance four times greater than that appropriate for viewing a "normal" picture or projection (to compensate for the focal length's being four times greater than normal), the normal projective geometry of the structured array will be received by the viewer. However, the extent of the surrounding field included in the picture remains the same, of course, that is, one-fourth of 
that normally included in unmagnified photographs. This means of obtaining normal projective geometry from the slide or picture prepared with optical magnification is mentioned here simply for purposes of further explication of magnification in general. In the study to be reported, subjects viewed the slides prepared with optical magnification from the shorter distance calculated as proper for viewing normal nonmagnified slides, thus maintaining the enlarged visual angles in the reflected optic array that had been effected by the magnifying lens system.

By uniformly enlarging the optic array by a factor equal to the power of magnification, the density of textural elements, the rate of change of density of these elements, and the slope of linear perspective per unit area within the retinal projection are lessened by a factor equal to the reciprocal of the power of magnification. Furthermore, the further truncation of the visual field which is coincident with the magnification excludes the nearer ground that normally specifies the relative distance to objects within the spatial layout (Lumsden, 1980; Purdy, Note 1). Holding other relevant parameters constant (such as distance of focus and lens design), the extent of visual surround or visual field that is transmitted is a decreasing, negatively accelerated function of the focal length or power of the optical lens system. The visual surround or field transmitted in the rectangular projection can be described in terms of height, width, or diagonal of the projection. For the present purposes, the visual angle subtended by the height of the rectangular projection would seem to be the most appropriate dimension, and it has therefore been utilized here. Needless to say, all three of the dimensions of the transmitted visual field are perfectly correlated.

There is ample empirical evidence demonstrating that truncation of the visual field reduces the perception of three-dimensionality. For example, it has frequently been reported that when a slanted surface is viewed through an aperture or a reduction screen, the plane of the slanted surface seems to be more parallel to the plane of the reduction screen than it actually is (Gibson, 1950; Gruber \& Clark, 1956). Another investigation utilized a visual field containing objects on a textured surface, with the field being truncated by being viewed monocularly through a picture frame, within a projected $35-\mathrm{mm}$ photographic slide (the visual field being truncated by its terminal boundaries), or through a peephole in a reduction screen (Hagen, Jones, Reed, 1978). The observers reported compression of perceived distance between the objects within the truncated visual field similar to the kind of perceptual compression experienced when visual fields that have been transformed by optical magnification are viewed. This experimental finding leads one to ponder the extent to which the "flatness effect" of optical magnification is due to the further truncation of the visual field coincident with optical magnification as opposed to the multiplicative enlargement of all visual angles retained within that visual field, that is, magnification per se. Purdy (Note 1) showed unequivocally that the transformation effected upon the various gradients of texture, relative size, and perspective correspond to those projected by a more frontal slant as well as a lesser radial distance between objects in the spatial layout. However, the grid pattern he magnified neither required nor permitted manipulation of the effects of coincident truncation. Although Hagen et al. did ably address the pertinence of truncation of visual field for the perception of radial distance as stated above, magnification was not a variable in that experiment. The purpose of the present investigation was to determine the relative effect of magnification per se and the further reduction of the visual field, normally coincident with optical magnification, upon the perception of radial distance in the depicted spatial layout.

\section{METHOD}

Using a single-lens reflex 35-mm camera, color slides were prepared of two vertical, white, $6-\mathrm{ft}(1.8-\mathrm{m})$ posts, $4 \mathrm{in} .(10.2 \mathrm{~cm}) \times$ 4 in. $(10.2 \mathrm{~cm})$, spaced $12 \mathrm{ft}(3.6 \mathrm{~m})$ apart. Both a normal lens of $50-\mathrm{mm}$ focal length and a telephoto lens of $200-\mathrm{mm}$ focal length were utilized, the latter effecting 4-power magnification. The distance from the camera to the point halfway between the posts was $90 \mathrm{ft}(27.4 \mathrm{~m})$. The line of sight was $10 \mathrm{deg}$ oblique to a line connecting the two posts, though no lines were visible when photographic slides were prepared. Under these conditions, the nearer post was almost $84 \mathrm{ft}(24.4 \mathrm{~m})$ from the camera, projecting a visual angle of $4 \mathrm{deg}$ through the normal lens and $16 \mathrm{deg}$ through the $200-\mathrm{mm}$ lens. The farther post was at a distance of about $96 \mathrm{ft}$ $(29.3 \mathrm{~m})$ from the camera and projected a visual angle of $3.6 \mathrm{deg}$ through the normal lens and $14.4 \mathrm{deg}$ through the $200-\mathrm{mm}$ lens. Inasmuch as the nodal point of the lens of the camera was positioned above the ground at one-half the height of the post $(3 \mathrm{ft}$, or $.9 \mathrm{~m}$ ) and the cylindrical axis of the lens system was parallel to the ground, the projection of the farther post was centered with respect to the projection of the nearer post. These slides, or modified versions of them, were back-projected onto a translucent projection screen which was viewed from the opposite side. The subject was positioned such that his or her eyes were $521 / 2$ in. $(1.3 \mathrm{~m})$ along a perpendicular from the center of the projected slide, which is the distance from which the same projective geometry would be received by the subject as was received by the film plane when it was originally exposed.

A $2 \times 2$ factorial design was employed utilizing two levels of magnification-normal (1-power) and 4-power-and two levels of truncation-the amount of truncation that is concomitant with the preparation of a $35-\mathrm{mm}$ slide utilizing a normal $50-\mathrm{mm}$ lens (retaining about $27 \mathrm{deg}$ of the visual surround vertically) and the greater amount of truncation which is concomitant with optically produced magnification in $35-\mathrm{mm}$ slides utilizing a $200-\mathrm{mm}$ lens (retaining only $6.9 \mathrm{deg}$ of the visual surround vertically). The displays were the following slides of the two posts: (1) taken with the normal lens and viewed from the distance required to receive the normal projective geometry of the visual field (condition normal/27-deg); (2) taken with the $200-\mathrm{mm}$ telephoto lens, producing 4-power magnification, and viewed from the same distance as the normal slide (condition 4-power/6.9-deg); (3) taken with 
normal lens and cropped in such a way as to exclude the same visual surround that was optically excluded by the use of the $200-\mathrm{mm}$ telephoto lens in the preparation of that experimental slide (condition normal/6.9-deg) (i.e., this normal, cropped slide and the 4-power magnified, optically truncated slide were isomorphic to a scale factor of 4); and (4) taken with the normal lens and viewed from such a decreased distance (13.25 in., or .3 m) as to effect the same projective geometry as did the slide that had been taken with the $200-\mathrm{mm}$ lens, except that the visual surround coincident with the use of this normal lens was retained (condition 4-power/27-deg).

The subjects were allowed to view the projected slides binocularly, and no structural constraints were employed to insure immobility of the head. It was necessary for the participant to look to his or her left at the post and the metric scale in the lab in order to standardize the use of units of measurement. This head movement assured a certain amount of motion perspective. The binocular, unrestrained conditions of viewing were deliberate and desirable because I wished to generalize from this study to the viewing of slides and other pictures under relatively unrestrained conditions.

Pilot studies for this investigation had demonstrated that somewhat extreme measures must be taken to eliminate sources of extraneous variation in the reports of perception of radial distance under these conditions. The major source of error to be minimized was the intersubject variability regarding the subjective length of a "foot" inasmuch as the participants were required to report the distance between the two posts in feet. To reduce this source of confounding variance, two procedures were employed: (1) One of the posts that appeared in the slide was actually present in the lab and was placed vertically against the wall with a large numbered scale (calibrated in feet and portions thereof) extending from its base to a point $15 \mathrm{ft}(4.6 \mathrm{~m})$ away at the base of the wall. This was pointed out to the subjects, who were also informed that the post was $6 \mathrm{ft}$ tall. The subjects were explicitly instructed to utilize this information in reporting the perceived distance separating the two posts in the projected slides. (2) The design of this experiment required that the actual distance between the two posts be the same for all the displays utilized. However, the participants were told that a pointed stake attached to the back side of each post extended below the bottom of the post and projected into the ground, so the posts were not sunk into the ground and were quite movable. They were also told that the posts might be at any of several distances apart in the slides that they would be viewing. Nevertheless, I was concerned that the use of a complete within-subjects design (with the same subject reporting on all four of the experimental slides) might well permit the detection of this "deception" and lead to an artifactual reduction in the reporting of perceptual differences. As a partial resolution of this potential problem, each subject judged only two of the experimental slide presentations. Thus, the design had both within-subject and across-subject judgments. In order to minimize the irrelevant variability that normally accompanies any across-subjects design, a standard comparison slide was prepared and judged by all participants; it was always presented between the two experimental presentations. This standard was prepared with a normal lens from a distance of only $221 / 2 \mathrm{ft}$ $(6.9 \mathrm{~m})$ along the same line of sight utilized in the preparation of the experimental slides. (Pilot work had revealed that when this slide was projected and viewed from the normal distance, the estimates of radial distance were more veridical and less variable than those obtained with the experimental slides.) The individual's report of radial distance for each of the experimental slides was standardized by expressing the estimate as a percentage of the radial distance that this same individual reported for this standard, common slide. Each of the experimental slide presentations appeared as often prior to the common standard slide as it did afterward. Thus, if judging the standard produced any differential transfer effects, this bias was fairly evenly distributed across the judgments of the experimental slide presentations.

The subjects were students of undergraduate psychology classes

Table 1. Design of Experiment and Results.

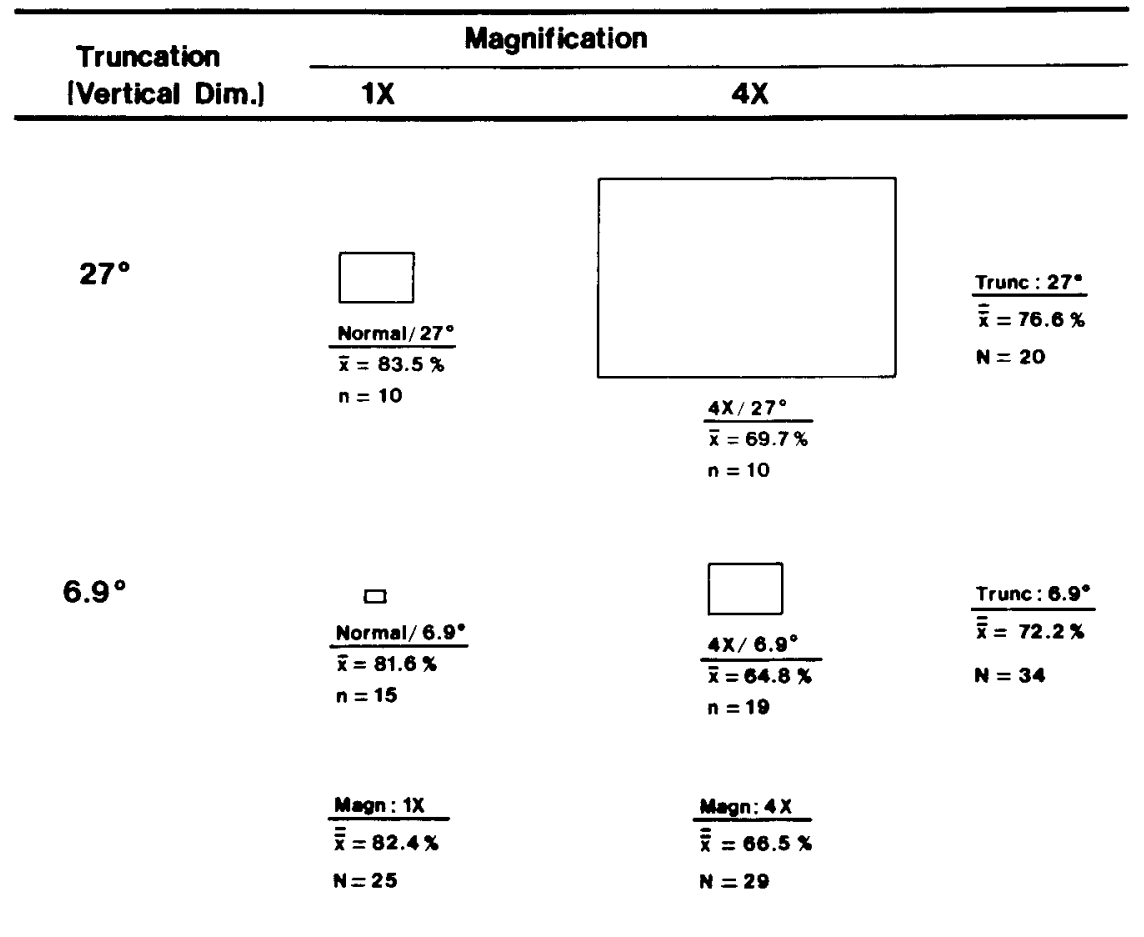

Note-Relative size of rectangles depict relative size of projections on the screen from the subject's station point. 
who served before studying the variables associated with distance perception. There were approximately an equal number of males and females, and vision was reported as normal or corrected to normal. With 27 subjects, each judging two experimental slides (and the one common, standard slide), a total of 54 experimental judgments were obtained and converted as described above (see Table 1). No experimental slide presentation was judged by fewer than 10 subjects.

\section{RESULTS AND DISCUSSION}

The basic data analyzed were the individual judgments of the radial distance between the two posts in the experimental slide presentations expressed as a percentage of the distance reported for the common slide for each individual participant. A two-way analysis of variance revealed that main effects for magnification were significant $[F(1,50)=7.46, p<$ .011 ; neither the truncation effects nor the interaction effects were significant.

It is important to specify adequately the conditions in which no significant effect of truncation was obtained because the results of Hagen et al. could lead one to expect a significant effect here. It will be recalled that the concern of this investigation was the effects of magnification produced by optical means, and that it is only this means of magnification that also further reduces or further truncates the visual surround in the process of magnification. The circumstances in which optical magnification is used are generally situations in which better visual differentiation is required within the optic array being reflected from objects that are relatively distant from the observer in units of object size. Consequently, the objects in the visual field project very small visual angles to the unaided eye, and the difference between the size of the projection of two distant objects of the same size that are radially separated is indeed minuscule. The curve in Figure 1 depicts the decreasing, negatively accelerated relationship between projected visual angle and viewing distance. Please note also that this figure shows that the visual angles subtended by these two posts at distances of about 84 and $96 \mathrm{ft}$ $(24.4$ and $29.3 \mathrm{~m})$ are 4.0 and $3.6 \mathrm{deg}$, respectively. Transformed by 4-power magnification, the visual angles become 16.0 and $14.4 \mathrm{deg}$. The resulting relative size gradient defined by these two enlarged projections corresponds to, or specifies, a radial distance between them of only $3 \mathrm{ft}(.9 \mathrm{~m})$ rather than the $12-\mathrm{ft}$ (3.7-m) radial distance presumably specified by the unmagnified array. Alternatively, Lumsden (1980) and Purdy (Note 1) provide formulas that permit the computation of this radial distance. I suspect, however, that the graph shown in Figure 1 permits better understanding of this optic transformation. When one realizes that the difference in size of the projected visual angles of these two posts without magnification is only .4 deg and that the angle separating the points where the posts intersect the ground in the pic-

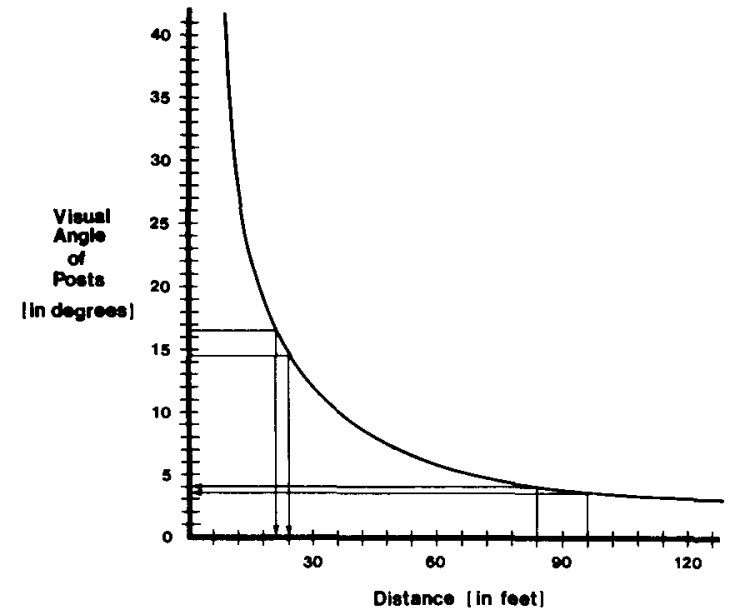

Figure 1. Curve depicting visual angle projected by the posts as a function of viewing distance. Also shows that distance specified by relative slze of the enlarged visual angles equals only 1 over the power of magnification times the real distance between the posts.

ture plane is only $.22 \mathrm{deg}$, it is not surprising to learn that the full extent of the distance between the two posts was not perceived. In fact, the average distance between the posts reported for the normal slide at normal distance was only $6.55 \mathrm{ft}(2 \mathrm{~m})$, which is only $54.6 \%$ of the real distance of $12 \mathrm{ft}(3.7 \mathrm{~m})$. Even our standard comparison slide yielded an average judgment of radial distance that was only $65 \%$ of the real distance. However, even with the more definitive, but equally truncated, arrays projected by the slides utilized in the study by Hagen et al. (1978), the average estimate of radial distance corresponded to only about $70 \%$ of the real distance between the reference marker and each of the triangles successively placed at different radial distances. It appears, then, from the Hagen et al. study and the other work referenced above, that truncation, however it may be produced, does reduce perception of radial distance even with monocular viewing, which minimizes perception of the frontoparallel projection surface. Therefore, our investigation did not require a comparison of the perception of radial distance under the greater truncation effected by magnification with the perception of radial distance under direct monocular viewing of the posts in the open field. Our most informative comparison is between perceived distance under the former condition (4-power/6.9-deg visual field) and perceived distance when viewing the projection of the same degree of magnification (4-power) but with only the reduction of visual surround that is normally effected by a normal lens (4-power/27-deg visual field). The point here is that underestimation of real radial distance between the distant posts is so great even in normally truncated slides (normal/27-deg) that little more compression of space can occur due to magnification and/or further truncation. 
As noted above, the immediate visual foreground normally present in untruncated viewing of an open field includes expansive perspective and textural gradients that may serve to specify relative distance to the objects in question. Hagen et al. (1978) suggested that if this information is excluded by truncation, the observer may tend to assume a shorter distance to the objects being viewed. Given a certain relationship between the projected size of two radially separated objects, if the observer fails to perceive the full distance to the nearer object, less radial distance between the two objects is geometrically specified. This line of reasoning also would require that the size of the objects be perceived as correspondingly smaller than they would if the real greater distance to them were perceived more veridically. The results of the Hagen et al. experiment that relate to this question were somewhat equivocal. Of particular pertinence here, however, is the fact that even with the extreme reduction in the visual field effected by the $2-\mathrm{mm}$ peephole, estimates of radial distance were not significantly reduced beyond those obtained with the use of a rectangular frame designed to effect the same reduction of visual surround as that in a normal 35-mm slide produced with normal lens. Thus, it would appear that, while truncation is a pertinent variable for the reduction of perceived radial distance, the results of Hagen et al. and the results of the investigation reported here suggest that the effects of truncation may be asymptotic at values equal to or less than the reduction produced by normal $35-\mathrm{mm}$ slides. In retrospect, the general outcome of the present study in regard to the main effects of truncation might have been predicted from the Hagen et al. study. However, this investigation has shown again that 4-power magnification can produce significant additional reduction in the perception of radial distance beyond the perceptual effects produced by the truncation of the field incurred in the preparation of an unmagnified slide. Furthermore, this additional truncation, coincident with optical magnification, does not produce interaction effects with magnification.

It must be acknowledged, however, that the reduced radial distance reported by the subjects from viewing the magnification presentations was of less magnitude than that expected on the basis of the lessened density and perspective gradients produced by the 4-power magnification. This finding of less reduction in the perception of radial distance than one would have predicted mathematically is consistent with the studies, reported above, that utilized reduction of viewing distance as the means of magnification of a depicted spatial array. Some of those authors have suggested the operation of a compensatory correcting process (Pirenne, 1970; Rosinski \& Farber, 1980).
While this study addressed the effects of optical magnification on the perception of radial distance projected in photographic slides (varying in magnification and truncation of visual surround), it seems reasonable to extend these findings to the conditions that prevail in viewing the actual visual field through a telescope. The telescope barrel alone without a lens system substantially reduces the visual surround retained in the transmitted array. Viewing through the lens system in the intact telescope further reduces the visual surround for the same reasons as does the lenticular preparation of our telephoto slide (4-power/ $6.9 \mathrm{deg}$ ). From the Hagen et al. study, we would expect that the truncation effected by the empty telescope barrel would effect a significant reduction in the perception of radial distance as compared with monocular nontruncated viewing of the field. If the results of our investigation can be extended to the use of a telescope, we would not expect the additional truncation of the visual field caused by the lens in the intact telescope to produce further reduction of perceived radial distance. However, the lens would be expected to produce further reduction in perceived radial distance as an effect of the reduced gradients of magnification, that is, if magnification is 4-power or more.

The lack of close correspondence, in this and related experiments, between the magnitude of radial distance perceived and the distance mathematically specified by the magnified gradients does seem to indicate that the perception of radial distance in pictorial displays is certainly not entirely determined by the density of gradients alone. However, one should be cautious about generalizing from this lack of correspondence (obtained with static projections of spatial layouts) to the effectiveness of such gradients under ecologically representative conditions of viewing. In the experiments related here, there is movement neither of the objects within the spatial layout nor of the observer through the layout. Consequently, data obtained from such conditions are inappropriate for addressing the relevance of the density of gradients of texture, relative size, or perspective for the normal perception of distance in the ecological surround (Gibson, 1979; Michaels \& Carello, 1981). It is quite unlikely that selective evolutionary advantage ever accrued to the definitive perception of radial distance in pictorial representations of spatial layouts. More currently, it is not clear how behavior could naturally and systematically be constrained by the definitive differentiation of radial distance between extremely remote objects through the use of optical magnification. Before direct interaction with the objects was possible, the distance between the observer and the objects would have decreased to the point at which magnification was no longer necessary. The movement involved in the reduction of this dis- 
tance, of course, would have produced motion perspective sufficient to more accurately specify the actual distance between the objects.

In summary, the present experiment on the effects of magnification on the perception of spatial relationships in a depicted spatial array is informative, but only for the viewing circumstances they approximate. To this extent, they bring an interesting addition to our knowledge about such viewing conditions. However, one should not generalize the relative inadequacy of gradients for veridically specifying distance in these circumstances to more ecologically representative situations.

\section{REFERENCE NOTE}

1. Purdy, W. C. The hypothesis of psychophysical correspondence in space perception. (Technical Information Series No. R60ELC56). Schenectady, N.Y: General Electric Co., 1960.

\section{REFERENCES}

Bartley, S. H., \& Adair, H. J. Comparisons of phenomenal distance in photographs of various sizes. Journal of Psychology, 1959, 47, 289-295.

Gibson, J. J. The perception of the visual world. Boston: Houghton-Mifflin, 1950.

Gibson, J. J. The ecological approach to visual perception. Boston: Houghton-Mifflin, 1979.
Gruber, H., \& Clark, W. C. Perception of slanted surfaces. Perceptual and Motor Skills, 1956, 6, 97-106.

HAGEN, M. A., JONES, R. K., \& REED, E. S. On a neglected variable in theories of pictorial perception: Truncation of the visual field. Perception and Psychophysics, 1978, 23, 326-330.

Lumsden, E. A. Problems of magnification and minification: An explanation of the distortions of distance, slant, shape, and velocity. In M. A. Hagen (Ed.), The perception of pictures (Vol. 1): Alberti's window, the projective model of pictorial information. New York: Academic Press, 1980.

Michaels, C. F., \& Carello, C. Direct perception. Englewood Cliffs, N.J: Prentice-Hall, 1981.

Pirenne, M. Optics, painting and photography. Cambridge: Cambridge University Press, 1970.

Rosinski, R. R. On the ambiguity of visual stimulation: A reply to Eriksson. Perception \& Psychophysics, 1974, 16, 259-263.

Rosinski, R. R., \& FARBer, J. Compensation for viewing point in the perception of pictured space. In M. A. Hagen (Ed.), The perception of pictures (Vol. 1): Alberti's window, the projective model of pictorial information. New York: Academic Press, 1980.

SMITH, O. W. Comparison of apparent depth in a photograph viewed from two distances. Perception and Motor Skills, 1958, 8, 69-81.

\section{NOTE}

1. A "normal" lens is one having a focal length roughly equal to the diagonal of the exposed film surface.

(Manuscript received January 7, 1982;

revision accepted for publication October 22, 1982.) 